\title{
Analysis of correlations between system structure and costs by structural criteria
}

\author{
Sebastian Maisenbacher, Stefanie Fink, Florian Behncke, Udo Lindemann \\ Institute of Product Development, Technische Universität München, Germany
}

\begin{abstract}
Structural system modeling uses matrixes like Design Structure Matrixes to model structures of various systems and uses analysis criteria to analyze and optimize these systems. Literature already discussed the application of structural criteria on certain system types. For example the analysis of design processes have been discussed by Kreimeyer (2009). Accordingly, this paper aims to identify and discuss correlations between product structures and costs. Therefore the Integrated Value Engineering (IVE) approach is considered as a starting point as it uses structural models to calculate cost values of products. To find correlations between product structures and costs an exemplarily use case is used. Preliminary structural criteria are identified, arranged and their applicability on different matrix types is discussed, to ensure that the exemplarily use case is analyzed in the right manner. Analysis results are directly compared to the cost values of the relevant elements of the product to identify correlations.
\end{abstract}

Keywords: Structural criteria, cost structures, integrated value engineering, cost management, structural analysis

\section{Introduction}

Matrixes, like design structure matrixes (DSM), domain mapping matrixes (DMM) and multiple domain matrixes (MDM), can be used to model dependencies of elements of a system. This can be for example the geometrical dependencies between components of a product. The matrixes support the practitioner in enhancing system understanding. Furthermore structural criteria can be used to analyze these matrixes to identify special characteristics of the underlying system structure which can have a special meaning for the system and supports system optimization. Existing literature already offers approaches to apply structural criteria and discusses the applicability on certain system structures like process structures or component structures.

In cost management the approach of integrated value engineering (IVE) uses matrixes to identify cost optimization potentials in products. Therefore the domains requirements, functions and components are modeled in a MDM and current costs as well as target costs are determined and assigned to the elements of all three domains. By a comparison of target and current costs optimization potentials can be identified and implemented later on. However, the applied matrixes, respectively system structure, is only used to calculate the cost values (Behncke et al. 2014). Accordingly to other structures we assume that these matrixes include further information which can be revealed by structural analysis. This information might be used to support product optimization in terms of costs and supports the implementation of cost potentials. 
Following these assumptions this paper has the objective to search for the existence of correlations between the structure of a system and its costs. Therefore structural metrics should be identified and classified on their applicability on different matrixes. Furthermore matrixes with additional cost values have to be analyzed.

Section 2 introduces fundamentals in structural system modeling and structural criteria to give an overview of possibilities in structural system analysis. Furthermore approaches in cost management are briefly discussed to introduce the approach of integrated value engineering, which basically combines product structures and costs. The results from literature allow to specify the research methodology for this publication (section 3 ). In section 4 first structural criteria are discussed and then applied on an exemplarily case study to determine direct correlations between the structure and its costs. Section 5 gives a conclusion and an outlook.

\section{Fundamentals in Cost Drivers and Structural Criteria}

This section starts with an introduction of structural modeling and structural analysis, followed by a brief introduction to cost management and to integrated value engineering to introduce relevant terms and methods for this publication.

\subsection{Structural modeling and analysis}

Structural modeling is basically used to enhance system understanding for the practitioner. It is based on the representation of systems as nodes and edges or respectively as elements and relations between elements. Elements of the same type are assigned to one domain. The relationship between one or two domains forms a subset which is equivalent to a matrix.

The systems can be depicted as graphs or in a matrix representation, whereas it is distinguished between three types of matrixes: A Design Structure Matrix (Eppinger and Browning, 2012) represents a subset of a single domain and a Domain Mapping Matrix (DMM) (Danilovic and Browning, 2007) represents the mapping of two different domains. A Multiple Domain Matrix (MDM) includes at least two domains and the affiliated subsets are represented by DSMs and DMMs (Lindemann et al. 2009). Several examples and industrial applications of structural modeling are given by Eppinger and Browning (2012).

\subsection{Structural criteria}

The matrix-based representations of a structure can be analyzed by different structural criteria, which are also mentioned as analysis criteria or structural metrics. Lindemann et al. (2009) and Kreimeyer (2009) give an extensive overview of structural criteria. Biedermann and Lindemann (2011) discuss requirements on the applicability of analysis criteria. For instance analysis criteria are mainly used for system analysis, but can also be helpful for system modeling. Furthermore the analyzed matrix types affect the applicability of structural criteria, as some cannot be applied on DMMs or require a symmetric matrix. Analysis in MDMs, which means analysis criteria are used cross 
domains, requires further interpretation, what is discussed in Kortler et al. (2010). An approach to optimize the structural analysis is introduced by Biedermann and Lindemann (2011). They also reveal correlations between the different structural criteria and show that some of the criteria have similar interpretations. For consistence in structural analysis it is suggested to select an optimal set of analysis criteria for the system. Additionally to the analysis with structural criteria, matrixes can be transferred in graph representations to achieve an easier accessible visualization for practitioners.

A specific focus of structural analysis is discussed in several literature. Exemplarily Kortler et al. (2010) discuss the application of structural criteria on the domains "components" and "employees", whereas Eben and Lindemann (2010) discuss the structural analysis of requirements. A detailed consideration of the structural analysis of processes is given by Kreimeyer (2009). All approaches have in common that the analysis is specified for one or more domains of structural models. The analysis of a system structure to draw conclusions on the costs is hardly comparable as the cost values are not modeled as elements in a domain. They can be seen as additional system properties which are linked to elements of a domain, what is used by the Integrated Value Engineering cost management approach.

\subsection{Approaches in cost management}

In global connected markets companies develop products in high competition. On the one hand high product quality and functionality and on the other hand lower prices in comparison to competitors increase the customer value. Approaches from cost management like target costing or value engineering support companies in achieving high customer oriented product functionality for low prices. Target costing focusses on deducing an optimal product's price from the market and determines target costs of a product and its components. Value engineering is used to optimize a product in terms of its customer value by comparison of the product's functionality with the components' costs to identify and implement optimization potentials (Cooper and Slagmulder, 1997; Ibusuki and Kaminski, 2007).

Basic ideas from target costing and value engineering are included in the relatively new approach of Integrated Value Engineering (IVE). IVE models products with the domains requirements, functions and components in a matrix, equivalent to a MDM. Then target cost are deduced and assigned to requirements and functions whereas current costs of components are estimated or deduced from data of comparable products. The subsets, respectively DMM, components fulfill functions and functions realize requirements are modeled and their relationships are weighted. These weighted matrixes are used to calculate the missing target and current cost values of the other domains. Therewith a target and current cost comparison is applicable on all three domains and supports in prioritizing elements for detailed consideration and deduction of value optimization potentials (see basic IVE model in Figure 1). Advantages in comparison to classical approaches is the possibility to find optimization potentials on all three product domains and therewith to analyze cause and effect of the product's elements on the product's value. Finally the implementation of the potentials is supported (Maisenbacher et al., 2013; Behncke et al., 2014; Maisenbacher et al., 2015). 

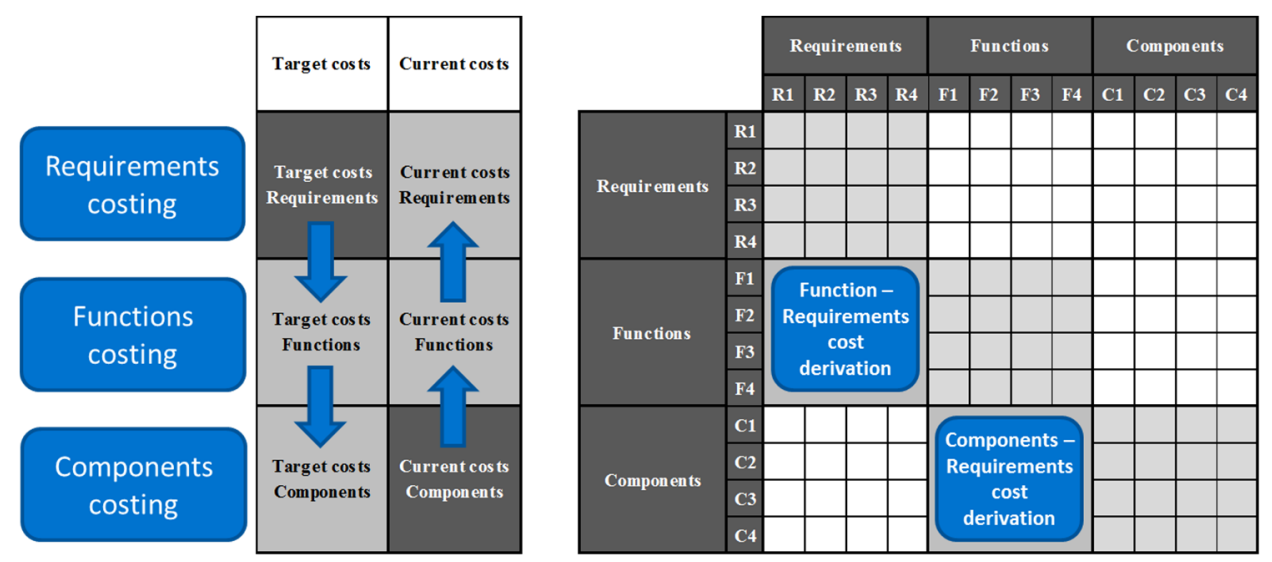

Figure 1. Integrated Value Engineering model (Maisenbacher et al., 2015).

\section{Research Methodology and Approach}

The IVE approach already combines structural modeling and costs. However, the matrixes are only used to calculate the missing cost values and not for an analysis of the product structure or for further optimization of the structure. To find correlations between system structures and system costs an existing IVE model is used. Therefore the IVE matrixes of a hair dryer are considered as a use case and are analyzed. Additionally further matrixes are modeled for the example and are analyzed. The analysis results are compared to the cost values of the elements of the hair dryer to reveal correlations. To ensure the correctness of the structural analysis structural criteria are deduced from literature and are structured. The applicability of structural criteria is also analyzed as especially the applicability on weighted matrices is hardly discussed in literature. The approach to find correlations between system structures and costs is illustrated in Figure 2.

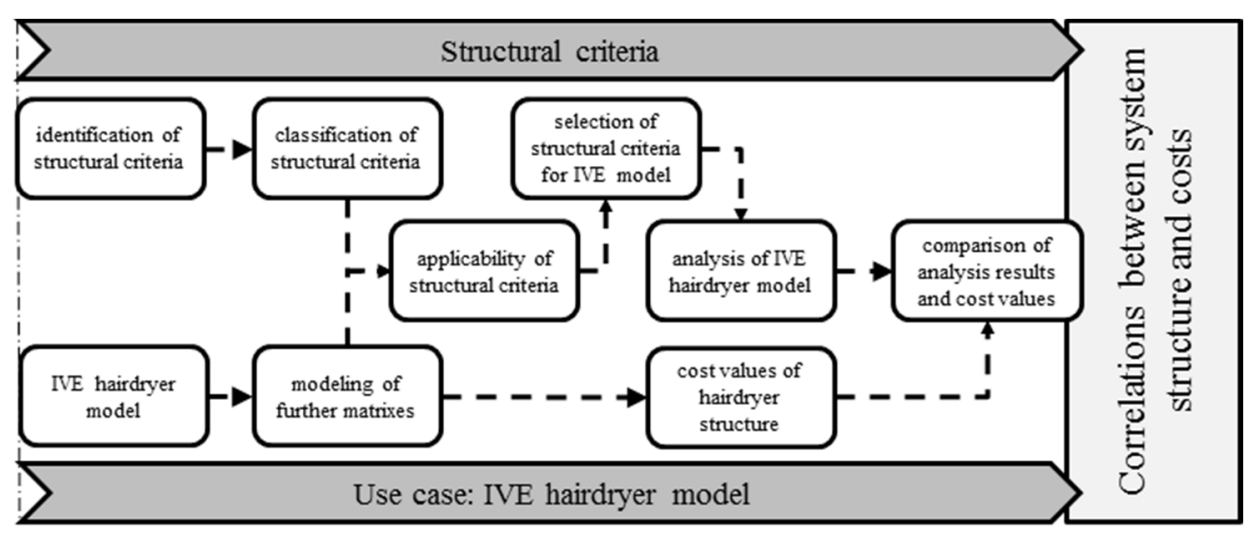

Figure 2. Approach to disclose correlations between structure and costs. 


\section{Correlations between Cost Structures and Structural Metrics}

In the following the approach from section 3 is presented in detail to prove if correlations between structural metrics and cost structures can be found. For this purpose, first a classification of structural metrics is described to get a better overview of the metrics and the correlations between them. The general applicability of these metrics in DSMs and DMMs is considered based on a practical example. The structural metrics are then applied on a hairdryer IVE model to analyze its structure and then compared with its cost values.

\subsection{Classification of Structural Metrics}

First, a classification of different structural metrics was developed. The metrics considered within this publication are taken from Kreimeyer (2009), Maurer (2007), Lindemann et al. (2009), Mathieson \& Summers (2009), Newmann (2003), Kim et al. (2011), Bellamy \& Basole (2013), Sosa et al. (2006), Wassermann \& Faust (1994), De Weck (2007), Sinha \& De Weck (2012), Biedermann et al. (2009) and Hossain \& Zulkarine (2011) who deal with structural complexity and network approaches. The 91 examined metrics are illustrated within a list which shows the definition and reference of each metric. Due to limited pages Figure 3 shows only an extract of the list of the structural metrics.

\begin{tabular}{|c|c|c|}
\hline Structural metric & Definition & Reference \\
\hline Activation of a cycle & $\begin{array}{l}\text { Number of nodes that are the first ones in a cycle (in a } \\
\text { triangularized DSM) }\end{array}$ & Kreimeyer (2009) \\
\hline Active Sum & $\begin{array}{l}\text { The active sum indicates the amount (and intensity) of all } \\
\text { outgoing edges of a node. }\end{array}$ & Maurer (2007), Kreimeyer (2009) \\
\hline Activity & $\begin{array}{l}\text { The activity of a node is composed by dividing its active sum by } \\
\text { its passive sum. The activity is a ratio for comparing different } \\
\text { nodes regarding their relative tendency toward an active or } \\
\text { passive behavior in a system. }\end{array}$ & $\begin{array}{l}\text { Maurer (2007), Kreimeyer } \\
\text { (2009), Lindemann et al. (2009) }\end{array}$ \\
\hline $\begin{array}{l}\text { All-pairs Shortest } \\
\text { Path (ASP) }\end{array}$ & $\begin{array}{l}\text { The sum of the values for the shortest path is the ASP. A higher } \\
\text { ASP indicates a more linear system (supply chain). The lower the } \\
\text { ASP is the more interconnections can be found. }\end{array}$ & Mathieson \& Summers (2009) \\
\hline Articulation node & $\begin{array}{l}\text { A node that exclusively links two groups of nodes. Only path } \\
\text { between two groups. }\end{array}$ & $\begin{array}{l}\text { Maurer (2007), Lindemann et al. } \\
(2009)\end{array}$ \\
\hline Average path length & Averages the path length between all nodes. & Newmann (2003) \\
\hline Banding & $\begin{array}{l}\text { Enhancement of partitioning, identification of elements that can } \\
\text { be executed in paraller or sequentially. }\end{array}$ & $\begin{array}{l}\text { Maurer (2007), Lindemann et al. } \\
\text { (2009) }\end{array}$ \\
\hline $\begin{array}{l}\text { Betweenness } \\
\text { centrality }\end{array}$ & $\begin{array}{l}\text { The measure indicates how often a node lies on the shortest } \\
\text { path between all combinations of pairs of other nods. Other } \\
\text { nodes are highly dependent on a node with a high betweenness. } \\
\text { Captures the possibility of a node to control and influence the } \\
\text { network }\end{array}$ & Kim et al. (2011) \\
\hline Bipartite density & $\begin{array}{l}\text { Percentage of the existing indirect relations and the number of } \\
\text { possible relations between domains }\end{array}$ & Kreimeyer (2009) \\
\hline $\begin{array}{l}\text { Bonacich power } \\
\text { centrality }\end{array}$ & $\begin{array}{l}\text { This measure gives a higher score to nodes that are directly } \\
\text { connected with other well-connected nods. }\end{array}$ & Bellamy \& Basole (2013) \\
\hline
\end{tabular}

Figure 3. Definition and reference of the first ten out of 91 structural criteria considered in this publication. 
For classification the structural metrics were divided into indicators and characteristics. An indicator can be understood as a kind of clue or basis of another structural metric. A characteristic, however, describes the attributes of an element. Afterwards, the characteristics were assigned to the appropriate indicators. Thereby, it was to determine which structural metric is the basis for another metric. Figure 6 shows a part of the classification of the structural metrics.

\subsection{Introduction to the exemplarily IVE model}

The use case for this work is the IVE model of a hairdryer. The reason for this selection is that a hairdryer is a relatively simple product. The information for the IVE model was acquired by using reverse engineering, which means to systematically decompose a product in its merest parts. For the creation of an IVE model the component domain as well as requirements and functions have to be considered. The elements of the components domain could be easily set by decomposing the physical hairdryer in its smallest elements. Functions have been modeled in using different types of functional models. A final hierarchical functional model was deduced in discussions with several engineering students and engineers. This hierarchical model allowed a proper selection of functions for the IVE model. The requirements have been deduced from the data sheet of the hairdryer and revised in several experiments and discussions. Finally 21 requirements, 24 functions and 38 components have been selected.

The current cost values of the components have been determined by searching similar components in spare parts catalogues and in the internet. If no similar components were found the current cost values have been estimated by consideration of the manufacturing effort for this component. Economics of scale have been discussed and also estimated. For target cost values a fixed cost reduction percentage has been considered. The two matrixes (one illustrated in Figure 4) to calculate the missing cost values have again been modeled by several engineering students and discussed with students and engineers.

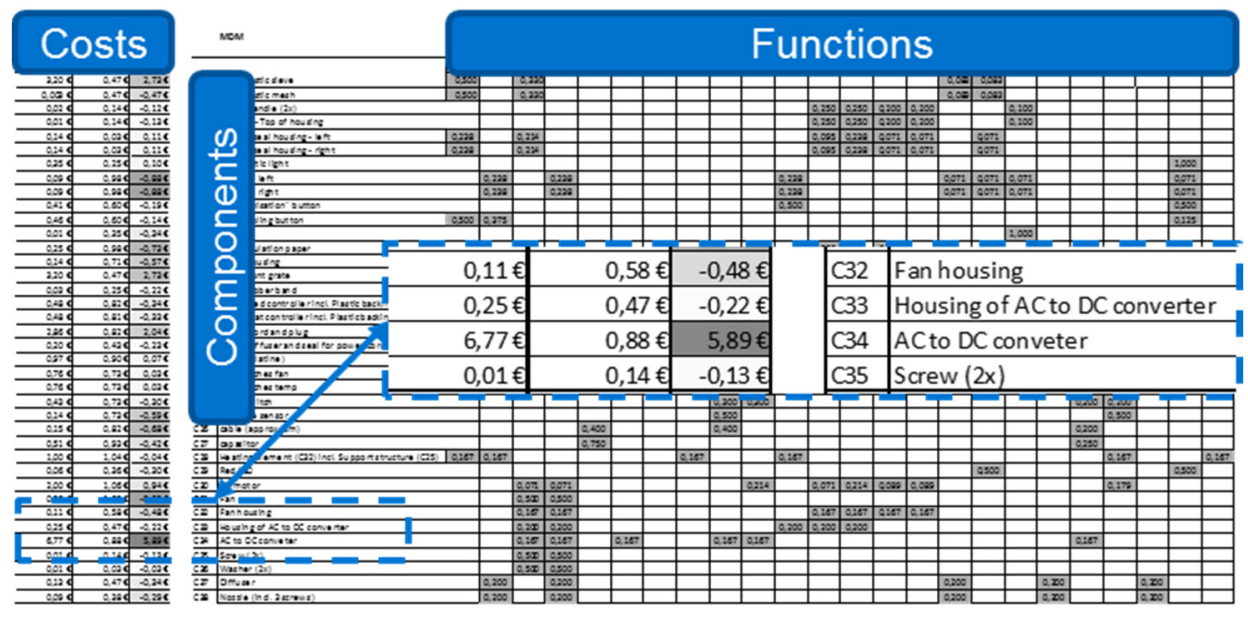

Figure 4. Part of the hair dryer IVE model with the AC to DC converter highlighted as the component with the highest cost optimization potential. 


\subsection{Applicability of Structural Metrics}

To examine the general applicability of the structural metrics in DSMs and DMMs, a structure analysis was conducted. The DMMs and DSMs used in the structure analysis are based on the IVE model of the hairdryer. In addition to the two weighted DMM of the IVE model further matrixes have been modeled or calculated to have all types of matrixes:

- DMM "Function fulfills requirement" (quantitative, weighted values from IVE model and same matrix reduced to qualitative values)

- DMM “Component fulfills function” (quantitative, weighted values from IVE model and same matrix reduced to qualitative values)

- DSM “Component is geometrically connected to component” (qualitative values)

- DSM "Component is functionally connected to component" (quantitative, calculated values from DMM "Component fulfills function")

In Figure 5 an extract of the Components-DSM which was created due to the geometric dependencies between the components is presented. The structural criteria from section 4.1 were now applied on the four matrixes. By analyzing the matrices it could be determined whether the application of the metric is possible or useful.

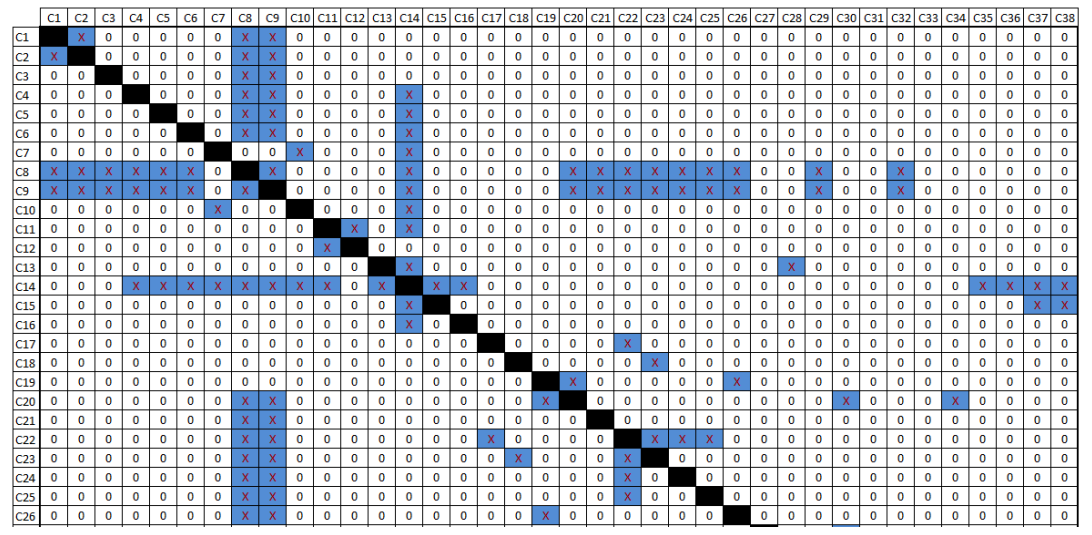

Figure 5. Components-DSM (geometric dependencies)

Thus, some of the structural criteria could be excluded because the application of them is not useful in matrixes which are DMMs, strongly connected or symmetrical. For example, it is not useful to determine the activity or passivity of an element in a DMM. In the classification of the structural criteria it was illustrated with different colors to what extent a criteria is applicable in the respective matrix. A white color, for example, means that the application of the criteria is not possible, a grey color indicates that the application is possible and useful and the application of a criteria marked with a light grey is possible, but not necessarily useful. For each of the analyzed matrixes the criteria were highlighted in the corresponding colors in the classification. Figure 6 shows an extract of the classification of the structural criteria. Herein the applicability of the metrics in the examined DMMs is visualized. 
Part V: Modelling functions and functionality of complex systems

\begin{tabular}{|c|c|c|c|}
\hline Indicator & Characteristic/Indicator & Characteristic/ Indicator & Characteristic/ Indicator \\
\hline Active sum & $\begin{array}{l}\text { Control-Flow Complexity (Boolean } \\
\text { Operators) }\end{array}$ & & \\
\hline \multirow[t]{12}{*}{ Number of nodes } & Number of unconnected nodes & & \\
\hline & Number of classes & & \\
\hline & Number of reachable nodes & & \\
\hline & Reachability of a node & & \\
\hline & \begin{tabular}{|l|} 
Number of independent sets \\
\end{tabular} & & \\
\hline & Degree distribution (+Activity, Passivity & & \\
\hline & $\begin{array}{l}\begin{array}{l}\text { Synchronisation/ distribution points } \\
\text { (+Number of edges) }\end{array} \\
\end{array}$ & & \\
\hline & Number of edges per node (+Number & (two different domains) & \\
\hline & \begin{tabular}{|l|} 
Degree of nodes \\
\end{tabular} & Centr ality of path (based on degree) & \\
\hline & Nodal degree & Isolierter Knoten (Nodal degr ee=0) & \\
\hline & \begin{tabular}{|l|} 
Locality \\
\end{tabular} & & \\
\hline & $\begin{array}{l}\text { Structural complexity metric } \\
\text { (+Number of edges) }\end{array}$ & & \\
\hline \multirow[t]{8}{*}{ Number of edges } & Cluster coefficient (local) & $\begin{array}{l}\text { Cluster coefficient (global) (+number } \\
\text { of nodes, Active sum) }\end{array}$ & \\
\hline & \begin{tabular}{|l|} 
Degree of connectivity \\
\end{tabular} & (two different domains) & \\
\hline & \begin{tabular}{|l|} 
Network/ Relational density \\
Module guality 1 flow of inform atio
\end{tabular} & (twn different dnmains) & \\
\hline & $\begin{array}{l}\text { Bipartite density (+Quantity of } \\
\text { indirect dependencies) }\end{array}$ & & \\
\hline & (Degree of non-planarity?) & & \\
\hline & \multirow[t]{3}{*}{ Path length } & Number of organis ational interfaces & \\
\hline & & \begin{tabular}{|l|} 
Max. path length \\
\end{tabular} & Diameter \\
\hline & & Average path length & \\
\hline
\end{tabular}

Figure 6. Applicability of structural metrics in DMMs

\subsection{Analyzing of an exemplarily cost structure}

The DSMs containing the relations between the components of the exemplary hairdryer were analyzed with the aim of determining correlations between the components costs and the structural metrics. A structure analysis was performed and the results were compared to the costs of the components. As can be seen in Table 1, the components with the highest criticality and those elements which are involved in the highest amount of indirect dependencies and feedback loops were among others identified.

Table 1. Exemplarily results of the structure analysis

\begin{tabular}{|l|l|}
\hline Structural metric & Components \\
\hline Highest criticality & $\mathrm{C} 17, \mathrm{C} 14, \mathrm{C} 19$ \\
\hline $\begin{array}{l}\text { Highest amount of indirect dependencies of length } \\
2\end{array}$ & $\mathrm{C} 17 \leftrightarrow \mathrm{C} 19$ \\
\hline Highest amount of feedback loops of length 2 & $\mathrm{C} 14, \mathrm{C} 17, \mathrm{C} 19$ \\
\hline $\begin{array}{l}\text { Highest amount of indirect dependencies of length } \\
3\end{array}$ & $\mathrm{C} 14 \leftrightarrow \mathrm{C} 17$ \\
\hline Highest amount of feedback loops of length 3 & $\mathrm{C} 17$ \\
\hline
\end{tabular}

The costs of the components $\mathrm{C} 17$ and $\mathrm{C} 19$ are quite high, whereas the costs of $\mathrm{C} 14$ are rather low. Furthermore, a triangularization and clustering of the matrix were conducted. 
It was not possible to detect any clusters which include components with similar costs. The arrangement of the components in the triangularized matrix did not provide a useful correlation with the costs of the components, too. The Locality of different elements, for example, was also considered. A clear difference between the components with high and those with low costs, however, could not be recognized. For instance, the component with the highest costs of the system has 26 directly connected elements, the component with the lowest costs has 24 connected elements. The other matrixes of the system (mentioned in 4.3) have been analyzed just as well. Altogether, significant and direct correlations between the results of the structure analysis and the costs of the components could hardly be identified.

\section{Conclusions and Outlook}

This paper had the objective to identify correlations between a system structure and the costs of the system. To ensure a comprehensive structural analysis, structural criteria have been extensively identified from literature and classified on their applicability for different matrix types. The IVE cost management approach has been discussed as an approach which already combines structural models and costs, but does not include structural analysis. Therefore an IVE model has been analyzed with the focus to identify correlations between the systems structure and its costs.

The structure analysis of the four matrixes of the hairdryer use case have not shown any significant correlations to the costs of the different elements. We assume that due to the preliminary detailed discussion of the structural criteria no errors in the application of the structural analysis are the reason for this result. Furthermore it is assumed that the most important structural criteria have been applied. Errors in the calculation of the cost values in the use case might be a source for errors in the comparison. For further work it is suggested to analyze further use cases and compare the results with the products costs.

However, we conclude that correlations between a system structure and its costs can be hardly directly disclosed by structural analysis. A reason could be that cost values are not directly included in structural models and are, for example in the IVE approach, considered as further system properties linked to the structure. According to literature it is suggested to consider structural analysis as a method which can analyze a system structure, for example in analyzing modularity of a product architecture by clusters, and to support optimization. An optimal product structure might have an indirect influence on the products' costs, for example a product with a higher degree of connectivity has more internal interface which increases its costs. An analysis which cost relevant steps in product development and which cost drivers can be supported by structure analysis and structural criteria might therefore for future work promising to find indirect correlations between system structure and costs. 


\section{References}

Behncke, F. G., Maisenbacher, S., Maurer, M., 2014. Extended Model for Integrated Value Engineering. Procedia Computer Science, 28, pp. 781-788.

Bellamy, M. A., Basole, R. C., 2013. Network analysis of supply chain systems: A systematic review and future research. Systems Engineering 16 (2), 235-249.

Biedermann, W., Kreimeyer, M., Lindemann, U., 2009. Measurement System to improve Data Acquisition Workshops. 11th International Design Structure Matrix Conference, DSM'09. Greenville, South Carolina, USA, 12.-13.10.2009.

Biedermann, W., Lindemann, U., 2011. Designing Consistent Structural Analysis Scenarios. $18^{\text {th }}$ International Conference on Engineering Design, ICED11. Copenhagen, Denmark, 15.18.08.2011.

Cooper, R., Slagmulder, R., 1997. Target Costing and Value Engineering. Productivity Press.

Danilovic, M., Browning, T. R., 2007. Managing complex product development projects with design structure matrices and domain mapping matrices. International Journal of Project Management, 25 (3), pp. 300-314.

De Weck, O. L., 2007. On the Role of DSM in Designing Systems and products for changeability. 9th International Design Structure Matrix Conference, DSM 07. Munich, 16.-18.10.2007.

Eben, K., Lindemann, U., 2010. Structural analysis of requirements-interpretation of structural criterions. The 12th International dependency and structure modelling conference. Cambridge, UK, 22.-23.07.2010.

Eppinger, S. D., Browning, T. R., 2012. Design structure matrix methods and applications. MIT press.

Hossain, S., Zulkarine, A. T., 2011. Design Structure of Scientific Software - a Case Study. Proceedings of the 13th International DSM Conference, DSM 2011.

Ibusuki, U., Kaminski, P. C., 2007. Product development process with focus on value engineering and target-costing: A case study in an automotive company. Int. J. Production Economics, 105, pp. 459-74.

Kim, Y., Choi, T. Y., Yan, T., Dooley, K., 2011. Structural investigation of supply chain networks: A social network analysis approach. Journal of Operations Management 29 (3), 194-211.

Kortler, S., Diepold, K. J., Lindemann, U., 2010. Structural Complexity Management using DomainSpanning Structural Criteria. 11 $1^{\text {th }}$ International Design Conference, Design 2010. Dubrovnik, Croatia, 17.-20.05.2010.

Kreimeyer, M. F., 2009. A Structural Measurement System for Engineering Design Processes. Diss. Technische Universität München.

Lindemann, U., Maurer, M., Braun, T., 2009. Structural Complexity Management: An Approach for the Field of Product Design. Berlin: Springer.

Maisenbacher, S., Behncke, F., Lindemann, U., 2013. Model for Integrated Value Engineering. Proceeding of the IEEE International Conference on Industrial Engineering and Engineering Management. Bangkok, Thailand 2013.

Maisenbacher, S., Stanglmeier, M., Behncke, F. 2015. Integrated Value Engineering - Adapted Approach to Assess different Concepts of a Jet Engine. $9^{\text {th }}$ Annual IEEE International Systems Conference, SysCon 15. Vancouver, BC, Canada, 13.-16.04.2015.

Mathieson, J., Summers, J., 2009. Relational DSMs in connectivity complexity measurement. 11th International Design Structure Matrix Conference, DSM 09. Greenville, 12.-13.10 2009.

Maurer, M. S., 2007. Structural Awareness in Complex Product Design. Diss. Technische Universität München.

Newmann, M. E. J., 2003. The Structure and Function of Complex Networks. SIAM Review 45 (2), 167-256.

Sinha, K., De Weck, O. L., 2012. Structural Complexity Metric for Engineered Complex Systems and ist application. 14th International Dependency and Structure Modelling Conference, DSM 12. Kyoto, 13.-14.09.2012. 
Sosa, M., Eppinger, S. D., Rowles, C. M., 2006. A Network Approach to Define Component Modularity. (Working Paper Series)

Wasserman, S., Faust, K., 1994. Social Network Analysis: Methods and Applications. Cambidge: Cambridge University Press.

\section{Acknowledgements}

We thank the German Research Foundation (Deutsche Forschungsgemeinschaft - DFG) for funding the project "cost analysis and optimization of mechatronic products by evaluating and designing product structures". 